\title{
Contribución del sector pecuario a la economía mexicana. Un análisis desde la Matriz Insumo Producto
}

\author{
Manuel Ernesto Sosa Urrutiaa , Francisco Ernesto Martínez Castañedaa* , J osé Antonio Espinosa Garcíab, \\ Germán Buendía Rodríguez'
}

\begin{abstract}
RESUMEN
La economía mexicana ha impulsado su crecimiento principalmente por las actividades terciarias, relegando a las actividades primarias a un último lugar de importancia. A pesar de esto, la producción primaria continua siendo muy importante para el desarrollo de otros sectores, ya que es un fuerte impulsor de la actividad económica; por ello, este trabajo se enfoca en analizar la contribución del sector pecuario en la economía, destacando sus aportes a la producción nacional y su relevancia en la demanda intermedia. Este aporte se analizó mediante la Matriz I nsumo Producto, la cual permitió conocer la oferta y demanda intersectorial, identificando los sectores más importantes en la economía. Se identificó que los principales encadenamientos productivos de las actividades pecuarias se encuentran en su interior, con una demanda de insumos del $69 \%$ para los sistemas ovino-caprino, 52 \% para el bovino, $46 \%$ para el porcino y el $45 \%$ para el avícola. Las ramas como el cultivo de oleaginosas y cereales siguen representando una proporción importante en su esquema de insumos. En lo que se refiere al multiplicador del producto, la explotación avícola (2.28) y la explotación porcina (2.24) fueron impulsores del desarrollo económico, debido al apalancamiento que representan para otros sectores de la economía, como oleaginosas, cereales y elaboración de alimentos para animales como los principales.
\end{abstract}

PALABRAS CLAVE: Ganadería, Política económica, Encadenamientos productivos, Leontief.

\begin{abstract}
The Mexican economy had boosted its growth primarily by tertiary activities, relegating the primary activities to the last place of importance. Despite this, primary production continues being very important for the development of other sectors, since it is a strong driver of economic activity; for this reason, this work focuses on analyzing the contribution of the livestock sector in the economy, highlighting their contributions to the national production and its relevance in the intermediate demand. This contribution was analyzed using the input output matrix, which allows to knowing the offer and demand cross-sector, identifying the most important sectors in the economy. Productive linkages were identified with inner livestock production activities and the input demand, ovine-caprine systems used $69 \%$, bovine used $52 \%$, swine $46 \%$ and poultry system used $45 \%$. Branches such as the cultivation of cereals and oilseeds remain a significant proportion in its pattern of inputs. In regards to the multiplier of the product, swine systems (2.28) and poultry (2.24) drove the economic development, because the leverage that represents for other sectors like oilseeds, cereals and animal feed industry.
\end{abstract}

KEY WORDS: Livestock, Economic policy, Productive linkages, Leontief.

Recibido el 1 de diciembre de 2015. Aceptado el 15 de febrero de 2016.

a Instituto de Ciencias Agropecuarias y Rurales Universidad Autónoma del Estado de México. Campus “El Cerillo", Piedras Blancas. Toluca. Estado de México. CP 50200. México.

b Centro Nacional de Investigación Disciplinaria en Fisiología y Mejoramiento Animal. Instituto Nacional de Investigaciones Forestales, Agrícolas y Pecuarias. Querétaro, México.

* Autor de correspondencia: femartinezc@uaemex.mx. 


\section{INTRODUCCIÓN}

El producto interno bruto (PIB) mexicano tuvo un crecimiento en términos reales del $69 \%$ durante el período de 1993 a 2014. Este comportamiento fue impulsado principalmente por las actividades terciarias y secundarias de la economía, las cuales, en promedio, contribuyeron en 58 y $36 \%$ respectivamente. Por su parte, las actividades primarias registraron $3 \%$ de participación en el PIB en el período señalado(1). Esta baja contribución podría ser atribuida a diferentes factores que van desde la apertura comercial en la que el país se encuentra inmerso desde 1994, así como la desgravación arancelaria unilateral ocurrida en 1986 y que favoreció la exportación de bienes industriales y la expansión de los servicios financieros, hasta la orientación de las políticas nacionales que relegan al sector agropecuario a un segundo plano en el orden de importancia como generador de riqueza en la economía ${ }^{(2,3,4)}$. De igual forma la contribución al empleo que realiza cada una de las mencionadas a la economía, presenta un panorama similar; para 2014, los servicios representaron el $62 \%$ de los empleos generados, seguido de la industria con $24 \%$ y el sector agropecuario con $14 \%^{(1)}$.

Las cifras anteriores claramente ubican a las actividades primarias en último lugar de importancia en el contexto general de la economía mexicana; sin embargo, su relevancia radica en la relación directa que éstas guardan con la seguridad alimentaria de la población, y sus relaciones con otras actividades en su rol de proveedores de insumos.

Bajo este contexto, durante el período de 1993 a 2014, la estructura del producto interno bruto agropecuario (PIBA) ubica al rubro agrícola como el más relevante, alcanzando una participación promedio de $60 \%$. Por su parte, la producción pecuaria aporta $32 \%$, seguido por las actividades de pesca, forestales y otros servicios relacionados que suman en su conjunto el $8 \%$ restante ${ }^{(1)}$. Debido a las relaciones intersectoriales que existen en la economía, algunas otras actividades, entre ellas las pecuarias, siven para incentivar a otros rubros ${ }^{(5)}$.

Es posible visualizar este tipo de relaciones mediante la metodología de la Matriz Insumo
Producto (MIP), la cual permite conocer la oferta y demanda intersectorial, identificando los sectores más importantes en la economía, además de su influencia sobre el resto de los sectores. La MIP expresa estas características mediante los encadenamientos productivos, los cuales se dividen en encadenamientos hacia delante (oferta de insumos) y encadenamientos hacia atrás (demanda de insumos) ${ }^{(6)}$.

Por lo anterior, el objetivo de este trabajo fue analizar la contribución del sector pecuario a la economía mexicana, resaltando su aporte a la producción nacional y su relevancia para el resto de sectores en la demanda intermedia.

\section{MATERIAL Y MÉTODOS}

Las cifras utilizadas en este estudio provienen en primer lugar, de las publicaciones oficiales del Instituto Nacional de Estadística y Geografía (INEGI), quien elabora la MI P que sirve de base para el análisis central del documento. La misma fuente ha sido utilizada para las cifras del PIB, tanto general como sectorial. Los datos concernientes a la producción se obtuvieron de los datos oficiales publicados por la Secretaria de Agricultura, Ganadería, Desarrollo Rural, Pesca y Alimentación (SAGARPA) a través del Sistema de Información Agroalimentaria y Pesquera (SIAP) y su base de datos denominado "Sistema de Información Agroalimentaria de Consulta" (SIACON).

\section{Modelo}

El presente estudio delimita la composición del subsector pecuario a las ramas de explotación bovina, porcina, avícola, ovina y caprina; definidas cada una por el Sistema de Clasificación Industrial de América del Norte, México(7). Se busca analizar su contribución mediante la MIP, la cual permite identificar e ilustrar el intercambio entre las distintas actividades $y$ productos que forman una economía ${ }^{(8)}$; su enfoque sectorial permite observar los efectos de cualquier variación en el valor bruto de la producción, analizando la oferta y demanda intersectorial ${ }^{(6)}$, esto es, a partir de los distintos 
requerimientos de insumo de una actividad. En la MIP se plasma la estructura técnica que permite establecer cuáles son las relaciones en que se involucra una actividad o producto, en términos de uso e intercambio con el resto del sistema económico (Cuadro 1).

Para estimar la importancia del subsector pecuario se utilizó la MIP 2012, elaborada por INEGI, la cual sirvió de base para realizar el cálculo de la matriz de coeficientes técnicos $(A)$, que se obtiene de dividir el consumo intermedio de cada sector entre el valor bruto de la producción del mismo $\left(\mathrm{Cl}_{\mathrm{ij}} / \mathrm{VBP}_{\mathrm{ij}}\right)$. Estos coeficientes reflejan el requerimiento directo de los insumos que cada rama necesita para generar su producto final o intermedio para los sectores con los que está interrelacionada.

$$
A=\frac{\sum_{i, j=1}^{n} a_{i j}}{\sum_{j=1}^{n} x_{j}}
$$

Donde $A=$ coeficientes técnicos,

$\sum_{i, j=1}^{n} a_{i j}$ : Consumo intermedio de las ramas $i$ demandado de las ramas $j$,

$\sum_{j=1}^{n} X_{j}$ : Valor bruto de la producción de las ramas ij

\begin{tabular}{|c|c|c|c|c|c|c|c|c|c|c|c|}
\hline & \multicolumn{4}{|c|}{ Demanda Intermedia } & \multicolumn{6}{|c|}{ Demanda Final } & \multirow{2}{*}{$\begin{array}{c}\text { VBP } \\
x_{i}\end{array}$} \\
\hline Productos & $\begin{array}{l}\text { Rama } \\
\text { A1 }\end{array}$ & $\begin{array}{l}\text { Rama } \\
\text { A2 }\end{array}$ & $\begin{array}{l}\text { Ram } \\
\text { a A3 }\end{array}$ & $\begin{array}{c}\text { Total } \\
\text { Consumo } \\
\text { Intermedio }\end{array}$ & $\begin{array}{c}\text { Consumo } \\
\text { Hogares }\end{array}$ & $\begin{array}{l}\text { Consumo } \\
\text { Público }\end{array}$ & FBKF & Existencias & Export & $\begin{array}{c}\text { Demanda } \\
\text { Total }\end{array}$ & \\
\hline Rama A1 & a11 & a12 & a13 & $\begin{array}{c}3 \\
\sum_{i, j=1} a 1 j \\
\end{array}$ & $\mathrm{C} 1$ & G1 & I1 & E1 & $\mathrm{Xn1}$ & Y1 & $\mathrm{X} 1$ \\
\hline Rama A2 & a21 & a22 & a23 & $\begin{array}{c}3 \\
\sum_{i, j=1}^{3} \mathrm{a} 2 \mathrm{j} \\
\end{array}$ & $\mathrm{C} 2$ & G2 & 12 & E2 & $\mathrm{Xn} 2$ & Y2 & $\mathrm{X} 2$ \\
\hline Rama A3 & a31 & A32 & a33 & $\begin{array}{c}3 \\
\sum_{i, j=1} a 3 j \\
\end{array}$ & C3 & G3 & 13 & E3 & $\mathrm{Xn3}$ & Y3 & X3 \\
\hline $\begin{array}{l}\text { Consumo } \\
\text { Intermedio }\end{array}$ & $\sum_{i=1}^{3} \mathrm{a} 1 \mathrm{j}$ & $\sum_{\mathrm{i}=1}^{3}$ & $\sum_{\text {ai3 }}^{3}$ & $\begin{array}{c}3 \\
\sum_{i, j=1} \text { aij }\end{array}$ & $\sum_{i=1}^{3} \mathrm{Ci}$ & $\sum_{i=1}^{3} \mathrm{Ci}$ & $\sum_{i=1}^{3} l i$ & $\sum_{i=1}^{3} \mathrm{Ei}$ & $\sum_{i=1}^{3} X_{n i}$ & $\sum_{i=1}^{3} Y_{i}$ & $\sum_{i=1}^{3} X i$ \\
\hline Remuneraciones & W1 & W2 & W3 & $\sum_{j=1}^{3} W i$ & & & & & & & \\
\hline $\begin{array}{l}\text { Impuestos } \\
\text { subsidios }\end{array}$ & $\mathrm{T} 1$ & $\mathrm{~T} 2$ & T3 & $\sum_{j=1}^{3} T i$ & & & & & & & \\
\hline Valor Agregado & VA1 & VA2 & VA3 & $\underset{j=1}{3}$ & & & & & & & \\
\hline $\begin{array}{l}\text { Valor Bruto de la } \\
\text { Producción }\end{array}$ & $\mathrm{X} 1$ & $\mathrm{X} 2$ & $\mathrm{X} 3$ & $\sum_{j=1}^{3} X_{i}$ & & & & & & & \\
\hline
\end{tabular}

Cuadro 1. Estructura de la matriz insumo producto

Fuente: elaboración propia con base a varios autores.

FBKF= formación bruta de capital fijo; VBP= valor bruto de la producción. 
Posteriormente se construyó una matriz identidad (I), cuyos elementos de la diagonal principal son todos iguales al número $1^{(9)}$. A ésta se le resta la matriz de coeficientes técnicos (A), obteniendo de esta forma la matriz de Leontief (IA).

$$
\begin{aligned}
& L=\left\{\begin{array}{lll}
1 & 0 & 0 \\
0 & 1 & 0 \\
0 & 0 & 1
\end{array}\right\}-\left\{\frac{\sum_{i, j=1}^{n} a_{i j}}{\sum_{i=1}^{n} X_{i}}\right\} \\
& \mathrm{L}=(\mathrm{I}-\mathrm{A})
\end{aligned}
$$

Hecho lo anterior, se obtuvo el determinante de la matriz de Leontief, cuyo valor debe ser mayor que cero, al igual que los elementos que la conforman no deben ser negativos, ya que de lo contrario los incrementos en la demanda final resultarán en reducciones de la producción.

$\mathrm{M}: \mathrm{X}(\mathrm{L})$

Donde: $M=$ determinante de la matriz de Leontief; $X=$ Escala mayor que cero $(X>0) ; L=$ matriz de Leontief.

De lo anterior, se obtuvo la matriz inversa de Leontief $[\mathrm{I}-\mathrm{A}]^{-1}$, que permite conocer las influencias recíprocas en el aparato industrial y calcular los requerimientos directos e indirectos de las ramas productivas $^{(10)}$.

Para obtener la matriz inversa se calculó la matriz de cofactores, la cual resultó de multiplicar $(-1)^{i+j}$ por el determinante de una sub-matriz conocida como menor, denotada por $\left[\mathrm{M}_{\mathrm{ij}}\right]$. El menor se calculó al eliminar de una matriz cuadrada de orden $n$, una fila y una columna; eso se hizo con cada uno de los elementos que conforman la matriz de Leontief, luego se obtienen varias sub-matrices cuadradas de orden $\mathrm{n}-1$.

A estas sub-matrices se les calculó su determinante, obteniendo de esta forma, los menores que sustituirán a cada uno de los elementos $A_{i j}$ y se multiplica $(-1)^{i+j}$ por cada menor, construyendo la matriz de cofactores. Los elementos de esta matriz se dividieron entre el determinante de la matriz de Leontief dando origen a la matriz inversa ${ }^{(11)}$.

$$
R=(I-A)^{-1}
$$

De los resultados obtenidos de la inversa de Leontief se obtienen los multiplicadores del producto y la oferta, así como los encadenamientos productivos que componen la estructura productiva de la economía.

\section{Encadenamientos productivos}

Son las relaciones y vinculaciones que tiene un sector con el resto de la economía a través de la demanda intermedia, y se clasifican en encadenamientos hacia atrás y encadenamientos hacia delante.

Los encadenamientos hacia atrás miden la capacidad de un sector de arrastrar a otros directamente ligados a él, por su demanda de bienes de consumo intermedio y estimulando a su vez la actividad de tales sectores. Por su parte, los encadenamientos hacia delante ocurren cuando un sector ofrece sus productos a otro sector, y que a su vez se convierten en insumos para éste, estimulando además a un tercer sector que provee de insumos al primero $^{(5)}$.

\section{Multiplicadores de la oferta y la demanda}

Los multiplicadores son definidos como el valor total de la producción, en todos los sectores de la economía, que es necesario para satisfacer un aumento en la demanda final de un determinado sector ${ }^{(10)}$. Estos multiplicadores pueden ser utilizados sobre la producción, el ingreso o el empleo ${ }^{(12)}$. En el caso de los multiplicadores del ingreso, reflejan el incremento en el ingreso como resultado del aumento en las inversiones, las cuales traen consigo el aumento en la demanda de bienes y servicios de un determinado sector ${ }^{(13)}$; por su parte, los multiplicadores del empleo reflejan la capacidad de una actividad de generar empleo, medido por el empleo requerido por un sector para crear una unidad de producto(14).

\section{Poder de dispersión}

El poder de dispersión es un indicador que señala cómo los efectos de una variación en la demanda de una determinada rama, se distribuyen en el resto de la economía(15). Este indicador da la 
pauta para saber si existe concentración de los efectos multiplicadores de una rama, ya sea sobre un número limitado de actividades, o si estos son ampliados al resto de la economía. Con esta metodología es posible planificar los estímulos que necesitan ser inyectados a un determinado rubro para generar mayor crecimiento en una economía(16).

Es importante señalar que diversos autores han elaborado metodologías que permiten tipificar a los sectores según la relevancia de sus encadenamientos, siendo las más conocidas las de Chenery y Watanabe ${ }^{(17)}$, Rasmussen $^{(18)}$ y Hirschman ${ }^{(19)}$. La categorización utilizada en este trabajo corresponde a la elaborada por Rasmussen, planteándose el análisis a partir de la siguiente fórmula:

$$
P D_{j}=\frac{\frac{1}{n} \sum_{i, j=1}^{n} a_{i j}}{\frac{1}{n} \sum_{1=1}^{n} \sum_{j=1}^{n} a_{i j}}
$$

Esta ecuación se construyó a partir de dividir los encadenamientos hacia atrás y delante, respectivamente, con el promedio de encadenamientos de la economía. De los resultados de la ecuación se tiene que los valores donde $\mathrm{PD} \geq 1$ representan aquellas actividades cuyos encadenamientos son mayores que el promedio de la economía; es así como se definen las siguientes categorías de dispersión:

Sectores clave: aquellos sectores cuyos encadenamientos hacia atrás y delante son mayores que el promedio de la economía, y que en consecuencia, alteraciones en este tipo de sectores afectan a la economía de forma significativa, tanto por la demanda intermedia como la demanda final.

Sectores estratégicos: aquéllos que poseen una baja demanda de insumos, pero que por el contrario suministran una gran cantidad al resto de la economía, es decir, que son afectados principalmente por la demanda final y no tanto por la demanda intermedia.

Sectores impulsores: poseen bajos encadenamientos hacia delante y son grandes demandantes de insumos, pudiendo proveer directamente la demanda final. En este caso la demanda intermedia posee una gran importancia, y por ello la economía en general podría verse

Cuadro 2. Principales encadenamientos hacia atrás de explotaciones ganaderas (participación porcentual)

\begin{tabular}{|c|c|c|c|c|}
\hline Ramas & $\begin{array}{l}\text { Explotación } \\
\text { bovina }\end{array}$ & $\begin{array}{l}\text { Explotación } \\
\text { porcina }\end{array}$ & $\begin{array}{l}\text { Explotación } \\
\text { avícola }\end{array}$ & $\begin{array}{l}\text { Explotación de ovinos y } \\
\text { caprinos }\end{array}$ \\
\hline $\begin{array}{l}\text { Cultivo de semillas oleaginosas, leguminosas y } \\
\text { cereales }\end{array}$ & 11 & 12 & 10 & 6 \\
\hline Explotación de bovinos & 52 & & & \\
\hline Explotación de porcinos & & 45 & & \\
\hline Explotación avícola & & & 44 & \\
\hline Explotación de ovinos y caprinos & & & & 69 \\
\hline \multicolumn{5}{|l|}{ Elaboración de productos lácteos } \\
\hline Otros cultivos & 9 & & & 4 \\
\hline Comercio & 6 & 7 & 7 & 3 \\
\hline Elaboración de alimentos para animales & 4 & 16 & 17 & 5 \\
\hline $\begin{array}{l}\text { Molienda de granos y de semillas y obtención de } \\
\text { aceites y grasas }\end{array}$ & & 3 & 4 & \\
\hline Resto de ramas & 18 & 18 & 18 & 18 \\
\hline
\end{tabular}

Fuente: Elaboración propia con base en la Matriz insumo producto 2012. INEGI. 
afectada por fenómenos coyunturales en este tipo de sectores.

Sectores independientes: tienen un nivel bajo de demanda de insumos y su producción está dedicada principalmente a satisfacer la demanda final. Los fenómenos coyunturales poco afectan al resto de sectores relacionados a estos ${ }^{(20)}$.

\section{RESULTADOS}

\section{Encadenamientos productivos}

Las cifras que se observan en el Cuadro 2, indican que la explotación pecuaria demandó la mayor cantidad de insumos al interior de sí misma, es decir, que el nivel de autoconsumo es sumamente elevado, destacando la explotación de ovinos y caprinos con el $69 \%$ de los insumos, seguido por la explotación bovina (52\%), explotación porcina (46 \%) y explotación avícola (45\%). De igual forma debe señalarse que la rama de "cultivo de semillas oleaginosas, leguminosas y cereales" es una de las más importantes para la producción pecuaria, ya que suministró en promedio el $10 \%$ de los insumos necesarios. También debe destacarse la rama "elaboración de alimentos para animales" que representó en promedio el $16 \%$ de los insumos necesarios para las explotaciones porcina y avícola. El resto de ramas representaron porcentajes poco significativos de forma individual, sin embargo, en su conjunto suman el $18 \%$ de los insumos necesarios.

Los encadenamientos hacia delante se presentan en el Cuadro 3, donde se evidencia el elevado nivel de autoconsumo que se genera por parte de las actividades pecuarias, ya que en promedio, representaron el $77 \%$ de los insumos ofertados. En este intercambio de bienes se incluye la comercialización de crías de ganado, así como individuos para la reproducción, entre otros. Bajo esta estructura de oferta de insumos, también se hace evidente la relación que existe entre los productos pecuarios con las actividades transformadoras, ya que destacan los rubros "matanza, empacado y procesamiento de carne de ganado, aves y otros animales comestibles" y

Cuadro 3. Principales encadenamientos hacia delante de explotaciones ganaderas (participación porcentual)

\begin{tabular}{|c|c|c|c|c|}
\hline Ramas & $\begin{array}{l}\text { Explotación } \\
\text { bovina }\end{array}$ & $\begin{array}{l}\text { Explotación } \\
\text { porcina }\end{array}$ & $\begin{array}{l}\text { Explotación } \\
\text { avícola }\end{array}$ & $\begin{array}{l}\text { Explotación de ovinos y } \\
\text { caprinos }\end{array}$ \\
\hline Explotación de bovinos & 59 & & & \\
\hline Explotación de porcinos & & 86 & & \\
\hline Explotación avícola & & & 72 & \\
\hline Elaboración de productos lácteos & 16 & & & \\
\hline Explotación de ovinos y caprinos & & & & 92 \\
\hline $\begin{array}{l}\text { Matanza, empacado y procesamiento de } \\
\text { carne de ganado, aves y otros animales } \\
\text { comestibles }\end{array}$ & 10 & 7 & 13 & 1 \\
\hline Curtido y acabado de cuero y piel & 3 & 2 & 4 & 0.3 \\
\hline $\begin{array}{l}\text { Fabricación de otros productos de cuero, } \\
\text { piel y materiales sucedáneos }\end{array}$ & 2 & 1 & 2 & \\
\hline $\begin{array}{l}\text { Preparación e hilado de fibras textiles, y } \\
\text { fabricación de hilos }\end{array}$ & & & & 2 \\
\hline Fabricación de telas & & & & 1 \\
\hline $\begin{array}{l}\text { Restaurantes de autoservicio, comida } \\
\text { para llevar y otros restaurantes con } \\
\text { servicio limitado }\end{array}$ & & 1 & 1 & \\
\hline Resto de ramas & 9 & 3 & 5 & 4 \\
\hline
\end{tabular}

Fuente: Elaboración propia con base en la Matriz insumo producto 2012. INEGI. 
"curtido y acabado de cuero y piel", a excepción de la explotación bovina cuyo segundo demandante es la "elaboración de productos lácteos". Las actividades anteriores ocuparon el segundo lugar de importancia en oferta de insumos que realizan las explotaciones pecuarias.

\section{Multiplicadores de la oferta y la demanda}

La MIP mexicana cuenta con 259 ramas de producción; de éstas, las más dinámicas en términos del efecto multiplicador que generan son las actividades industriales, principalmente las relacionadas a tecnología, sobresaliendo la fabricación de equipos de audio y video, la cual exhibe un multiplicador del producto de 4.32; por el lado de la oferta, la rama de fabricación de productos derivados del petróleo y del carbón posee el mayor multiplicador, alcanzando 21.9. Al ubicar a cada una de las actividades de origen pecuario, la más relevante fue la explotación avícola con un multiplicador de la demanda de 2.28 , seguido por la explotación porcina 2.24. Por el lado de la oferta, los multiplicadores pecuarios más relevantes fueron la explotación bovina con 1.70 y la explotación avícola con 1.39 (Cuadro 4).

\section{Poder de dispersión}

Esta clasificación ubicó las actividades de explotación porcina y explotación avícola como sectores impulsores, ya que poseen un fuerte arrastre al proveer una gran cantidad de insumos a la industria; las actividades de explotación bovina y de explotación ovina y caprina se ubicaron como sectores independientes, ya que su producción está orientada principalmente como consumo final y no como productos intermedios. Otras actividades relacionadas a la producción pecuaria por el lado de la demanda, como el cultivo de oleaginosas y el curtido y acabado de cuero y piel por el lado de la oferta, se ubicaron entre los sectores estratégicos e impulsores respectivamente (Cuadro 5).

\section{DISCUSIÓN}

Los resultados descritos en los cuadros anteriores pueden variar en cada región del país, ya que cada una posee características económicas particulares que favorecen el desarrollo de ciertas actividades. La explotación pecuaria se concentra en la región del altiplano, centro norte, región noroeste y región occidente ${ }^{(21)}$. Este tipo de actividades son clasificadas como de mucha importancia para el desarrollo de las economías regionales, ya que poseen una capacidad de arrastre significativa ${ }^{(22)}$ al estar relacionadas a la alimentación, generando una contribución a la economía no solo desde el ámbito de la producción por sus encadenamientos, sino también por su demanda final(23).

Al analizar las cifras de las actividades pecuarias desde la MIP mexicana del 2012, se observó que la demanda intermedia que éstas generaron, representó el $2.1 \%$ del valor total de la producción en la economía, mientras que en la demanda final de alimentos, que alcanzó los MXN\$176,847 millones, el $30.2 \%$ correspondió a los productos

Cuadro 4. Multiplicadores de la oferta y demanda con base en la Matriz insumo producto 2012

\begin{tabular}{lcccc}
\hline \multicolumn{1}{c}{ Ramas } & $\begin{array}{c}\text { Multiplicador } \\
\text { demanda }\end{array}$ & $\begin{array}{c}\text { Ranking } \\
\text { demanda }\end{array}$ & $\begin{array}{c}\text { Multiplicador } \\
\text { oferta }\end{array}$ & $\begin{array}{c}\text { Ranking } \\
\text { oferta }\end{array}$ \\
\hline Fabricación de equipo de audio y de video & 4.3221 & 1 & 3.3227 & 30 \\
Fabricación de productos derivados del petróleo y del & 2.5243 & 41 & 21.9028 & 1 \\
carbón & 1.9107 & 133 & 1.7091 & 72 \\
Explotación bovina & 2.2419 & 83 & 1.1644 & 136 \\
Explotación porcina & 2.2820 & 70 & 1.3974 & 98 \\
Explotación avícola & 1.4421 & 230 & 1.0856 & 158 \\
Explotación de ovinos y caprinos & & & \\
\hline
\end{tabular}

Fuente: Elaboración propia con base MIP 2012. INEGI. 
Cuadro 5. Categorización de actividades pecuarias y principales encadenamientos según poder de dispersión

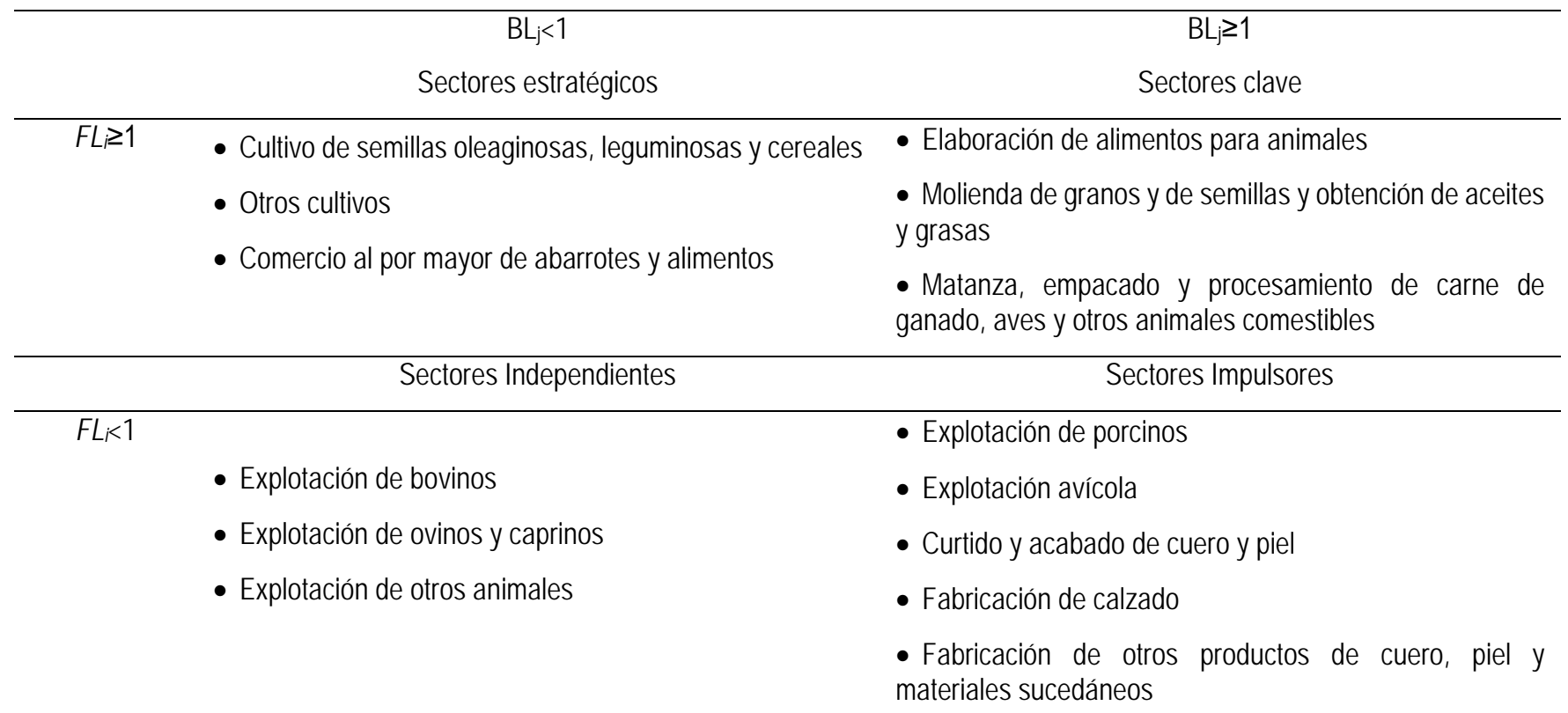

Elaboración propia con base a cálculos desde la Matriz insumo producto 2012. INEGI y Sobarzo 2011.(15)

pecuarios. Esta demanda sectorial se compuso principalmente por el consumo privado que ocupó el $68 \%$, seguido por las variaciones en los inventarios con $23 \%$, las exportaciones $14 \%$ y las importaciones $2 \%(1)$.

De igual forma la MIP 2012 indicó que en cuanto a la generación de valor agregado y empleo, las actividades pecuarias representaron el 27 y $12 \%$ respectivamente del total generado por las actividades primarias ${ }^{(1)}$.

Los datos mencionados dan paso para analizar otros usos de la MIP, ya que ésta también es utilizada como herramienta de planificación para la toma de decisiones en las políticas públicas, principalmente a través de la estimación de impactos por determinadas medidas a un sector ${ }^{(14)}$. Debe señalarse que la mayoría de estos análisis son realizados a partir de los encadenamientos hacia atrás, que son una herramienta muy importante para las políticas industriales ${ }^{(24)}$. Los encadenamientos productivos pueden ser utilizados para calcular los efectos de determinadas inversiones en la economía, reflejándose en cambios en la demanda final, lo cual permite evaluar escenarios probables para políticas que fomenten el crecimiento económico. En este estudio no se detectó un cambio en las inversiones en producción, pero sí una disminución en las preferencias de consumo. Este enfoque se ha realizado sobre excedentes de producción petrolera ${ }^{(12)}$, la industria turística(25), emisiones de $\mathrm{CO}_{2}{ }^{(5)}$ y empleo ${ }^{(13)}$ entre otros; sin embargo, hasta el momento son pocos los estudios publicados que abordan los impactos generados por las actividades pecuarias en la economía, ya que los existentes analizan especialmente a las actividades agrícolas como centro de la discusión, ubicando al resto de sectores en un segundo plano de discusión.

La información presentada para cada subsector pecuario permite detectar la importancia que cada actividad ganadera representa para la economía de México. Como una actividad demandante de insumos, la explotación avícola es la que presenta el multiplicador mayor, y por lo tanto la que tiene más capacidad de generar inversiones. Las producciones de carne de ave y de huevo, han tenido un comportamiento muy dinámico, con tasas de crecimiento de 182.3 y $126.5 \%$ durante el periodo 
de 1980-2010, respectivamente ${ }^{(26)}$. La combinación de estas tasas de crecimiento con el valor del multiplicador de 2.28, indica que cada año la producción avícola detona desarrollo en los sectores que la abastecen de insumos, como es el caso de los granos, oleaginosas, y el sector de elaboración de alimento para ganado.

La explotación de porcinos también presenta un multiplicador de la demanda similar a la explotación avícola; sin embargo, el comportamiento de dicho sector ha sido muy errático, demostrando graves crisis durante la década de los años ochenta y un crecimiento sostenido en las décadas subsecuentes ${ }^{(27)}$.

La explotación bovina se sitúa en una posición intermedia, dado que su crecimiento ha sido moderado, la producción de carne de bovino creció a una tasa de 40 \% y la producción de leche creció a una tasa de $43.9 \%$ durante el periodo 1980 $2010^{(26)}$ y su multiplicador de la demanda de menos de $2 \%$. La explotación de ovinos y caprinos por su parte, representa un aporte al valor de la producción de menos del $3 \%$ del valor total de la producción pecuaria(28), que combinado con el bajo valor del multiplicador de la demanda, tienen un bajo impacto en la económica.

Al analizar el impacto que cada una de las actividades pecuarias tienen como oferentes de insumos a otros sectores de la economía, la explotación de bovinos es la que presenta el multiplicador más alto, principalmente porque la leche de bovino que se consume en México sufre un proceso de transformación y se convierte en un insumo para la elaboración de productos lácteos, los cuales cada vez se demandan con mayor valor agregado(29).

En el presente análisis, la posición de cada actividad pecuaria en su capacidad de dispersión, tanto hacia los sectores abastecedores de insumos como a los demandantes de estos, ubicó a la explotación avícola y la de porcinos como sectores impulsores, y a la explotación de bovinos, ovinos y caprinos como sectores independientes. Dicho resultado no fue posible comparar, porque los estudios que han realizado un análisis para detectar sectores clave dentro de la economía mexicana ${ }^{(30,31)}$, solo hacen referencia al sector primario.
Otro uso hasta ahora poco aplicado de la MIP es construcción de matrices multiregionales, que relacionan el comercio y la producción entre los países $^{(32)}$. Este tipo de matrices permiten identificar el componente producido localmente y el importado con facilidad, debido a que las relaciones productivas son reflejadas en las exportaciones para el país proveedor de insumos y en las importaciones para el país que elabora el bien final. En los países de América Latina existen grandes limitaciones para desarrollar este tipo de matrices, debido principalmente a la escasez de información regionalizada; sin embargo, Brasil y México han logrado incursionar en esta dinámica internacional, siendo que el primero exporta bienes de consumo intermedio, por lo que posee una integración multiregional hacia delante, al ofertar a otros países para producir; el segundo lo hace mediante la exportaciones de bienes finales(32), es decir, su orientación en el mercado internacional lo ubica en una integración hacia atrás, ya que demanda de otros países bienes intermedios para ser ensamblados y producir un bien final. Desafortunadamente las actividades de origen pecuario no figuran dentro de este esquema multiregional, ya que se ha favorecido principalmente a las actividades netamente industriales. A pesar de esto no debe menospreciarse la capacidad de las actividades pecuarias en este contexto, ya que ante la dinámica que exhiben los mercados internaciones, es importante para los países diversificar el tipo de productos que exportan, por lo que se debe promover a su interior una adaptación sectorial ${ }^{(30)}$ que permita a otros productos posicionarse dentro de la oferta internacional; ello sin duda escalaría al país hacia nuevos niveles de competitividad en este tipo de mercados. Estos hechos llaman a una reflexión sobre el potencial y el rol que ejecutan algunos sectores de la economía, por lo que futuras investigaciones podrían ahondar en este tema, y así ampliar el conocimiento sobre el verdadero aporte que las actividades pecuarias realizan a la economía y en el comercio internacional.

\section{CONCLUSIONES E IMPLICACIONES}

El estudio reflejó que entre las actividades pecuarias las explotaciones avícola y porcina poseen 
una mayor capacidad de incidir en las decisiones de inversión en la economía, debido a la dispersión de sus efectos de multiplicadores, principalmente en las actividades avícolas, por lo que representan sectores con potencial de rescate de la dinámica productiva. Por su parte, las explotaciones bovina, ovina y caprina son rubros que poseen un gran potencial para incentivar la demanda final en una economía desacelerada. Las actividades pecuarias son fuertes impulsores del desarrollo de los cultivos de oleaginosas, la industria procesadora de alimentos y del sector servicios, evidenciando su importancia intersectorial. El potencial de las actividades pecuarias para generar nuevas inversiones permite no solo discutir su contribución en el ámbito productivo nacional, sino también abre una ventana hacia mercados externos, con lo cual el país puede posicionarse como un proveedor de insumos pecuarios en modelos especializados de comercio regionales.

\section{AGRADECIMIENTOS}

Se agradece al programa de becas del Instituto Interamericano de Cooperación para la Agricultura (IICA) y el Consejo Nacional de Ciencia y Tecnología de México (CONACYT) por la beca otorgada a Manuel Sosa; al Instituto Nacional de Investigaciones Forestales, Agrícolas y Pecuarias (INIFAP) y a la Universidad Nacional Autónoma del Estado de México a través del Instituto de Ciencias Agrícolas y Rurales por su apoyo a esta investigación.

\section{LITERATURA CITADA}

1. INEGI. Instituto Nacional de Estadística y Geografía. Sistema de cuentas nacionales. http://www.inegi.org.mx/est/contenidos/ proyectos/cn/. Consultado 17 Sep, 2015.

2. Sainz E. "Norteamérica busca su futuro más allá del NAFTA". El País. Enero 2014. http://internacional.elpais.com/internacional/ 2014/01/18/actualidad/1390070405_038418.html. Consultado 28 Ago, 2015.

3. Yunez-Naude A, Barceinas F, Soto RG. El campo mexicano en los albores del siglo XXI. El Nvo Mil Mex 2000;4:183-213.

4. Hernández MS. El sector agrícola en México, un análisis de política pública para el caso del maíz. (1994-2008) [tesis licenciatura]. Oaxaca, México: Universidad del Mar; 2010.

5. Schuschny AR. Tópicos sobre el modelo de insumo-producto: teoría y aplicaciones. Reunión de trabajo sobre modelización, matrices insumo-producto y armonización fiscal. Santiago de Chile. Chile. 2005.

6. Hernández G. Matrices insumo-producto y análisis de multiplicadores: Una aplicación para Colombia. Eco Insti 2012;26(14):203-221.

7. INEGI. Instituto Nacional de Estadística y Geografía. Sistema de Clasificación Industrial de América del Norte, México. SCIAN 2013. http://www.inegi.org.mx/est/contenidos/proyectos/SCI AN/present acion.aspx?_file=/est/contenidos/proyectos/SClAN/doc/scian2013. pdf

8. Soza $S$, Aroca P. Análisis y simulaciones en base a las matrices insumo-producto de la comuna de Punta Arenas para los años 2003 y 2007. Magallania 2012;2(40):113-127.

9. Weber JE. Matemáticas para administración y economía. New York. USA: Harper \& Row. Publishers Inc.; 1984.

10. Miller ER, Blair PD. Input-output analysis: Foundations and extensions. 2nd ed. New Jersey. USA: Prentice Hall Inc; 1985.

11. Aguilera R. Matemáticas 3 para ciencias económicas. 3a ed. La Libertad. El Salvador: UCA Editores; 1998.

12. Fuentes NA, Cárdenas, A. Evaluación del impacto de alternativas de utilización de los excedentes petroleros sobre la economía mexicana. Una aplicación del modelo insumo-producto. Econ Mex 2010; XIX(2):379-399.

13. Castaingts $\mathrm{J}$. Crecimiento y distribución del ingreso en una economía pequeña y abierta. Comer Ext 1998;48(6):431-442.

14. King A, Parra J C, Pino O. National economy 2008: A look from the perspective of the linkages for employment. Eur Sci J 2012;8(19):118.

15. Sobarzo FHE. Modelo de insumo - producto en formato de matriz de contabilidad social. Estimación de multiplicadores e impactos para México. Econ Mex 2011;20(2):237-280.

16. Iraizoz B. ¿Es determinante el método en la identificación de los sectores clave de una economía? Una aplicación al caso de las tablas input-output de Navarra. Estad Esp 2006;48(163):551-581.

17. Chenery HB, Watanabe T. International comparisons of the structure of production. Econométrica 1958;26(4):487-521.

18. Rasmussen N. Studies in Inter-sectoral relations. Am Econ Rev 1957; 7(3):432-435.

19. Hirschman AO. The strategy of economic development. New Heaven, USA: Yale University; 1958.

20. Soza ASA. Análisis estructural input-output: antiguos problemas y nuevas soluciones [tesis doctorado]. Oviedo, España: Universidad de Oviedo; 2007.

21. Castillo SSH. Evaluación del desempeño económico de la región sur de México [tesis maestría]. Coahuila, México: Universidad Autónoma de Coahuila; 2012.

22. Universidad Autónoma de Coahuila. Matriz insumo producto de la región centro occidente. www.centroccidente.org.mx/ matrizinsumoproducto/Reporte_RCO.docx. 2008.

23. Khem RS, PingSun L, Stuart TN. Accounting for the linkages of agriculture in Hawaii's economy with an input-output model: A final demand-based approach. Ann Regional Sci 1999;33:123-140.

24. Guerrero LC. La manufactura mexicana, diagnóstico de su estructura y programas locales de apoyo: prácticas, logros y 


\section{CONTRIBUCIÓN DEL SECTOR PECUARIO A LA ECONOMÍA MEXICANA}

pendientes hacia una política industrial nacional. Naciones Unidas. México. 2012

25. Marquina BS. Comparación y articulación interna de la actividad económica del sector turístico a partir de la matriz insumo producto turística de México (MI PTM-2003) basada en el enfoque de Cuenta Satélite del Turismo (C.S.T.) [tesis doctorado]. Madrid: Universidad Autónoma de Madrid; 2014.

26. Brambila-Paz JJ, Martínez-Damián MA, Rojas-Rojas MM, PérezCerecedo V. El valor de la producción agrícola y pecuaria en México: fuentes del crecimiento, 1980-2010. Rev Mex Cienc Agric 2014;4(5):619-631.

27. Bobadilla E, Espinoza A, Martínez F. Dinámica de la producción porcina en México de 1980 a 2008. Rev Mex Cienc Pecu 2010; 1(3):251-268.
28. Cruz M, Polanco M. El sector primario y el estancamiento económico en México. Probl Desarro 2014;45(178):9-33.

29. Blanco M. La activación de los SIAL vía el agroturismo: análisis del potencial de articulación en cuatro territorios queseros de América Latina. Agroalimentaria 2012; 18(34): 123-131.

30. Ocegueda H, Castillo P, Varela L. Crecimiento regional en México: especialización y sectores clave. Poblac Desarro 2009;40(159):61-84.

31. Figueroa O. Identificación de los sectores clave de la economía mexicana. Invest Cienc 2015;23(65):48-58.

32. Durán LJ, Zaclicever D. América Latina y el Caribe en las cadenas internacionales de valor. CEPAL Rev 2013;(124):1-66. 